\title{
Adaptive Detection of Instabilities: An Experimental Feasibility Study.
}

\author{
R. Rico-Martínez ${ }^{1,2 *}$, K. Krischer ${ }^{2}$, G. Flätgen ${ }^{2}$, \\ J.S. Anderson ${ }^{3}$ and I.G. Kevrekidis ${ }^{2,3}$ \\ ${ }^{1}$ Instituto Tecnológico de Celaya, Depto. de Ingeniería Química, \\ Celaya, Gto. 38010 México \\ ${ }^{2}$ Fritz-Haber-Institut der Max-Planck-Gesellschaft, \\ Faradayweg 4-6, 14195 Berlin, Germany \\ 3 Department of Chemical Engineering, Princeton University, \\ Princeton, NJ 08544 U.S.A.
}

October 25, 2018

\begin{abstract}
We present an example of the practical implementation of a protocol for experimental bifurcation detection based on on-line identification and feedback control ideas. The current experimental practice for the detection of bifurcations involves a cumbersome approach typically requiring long observation times in the vicinity of marginally stable solutions, as well as frequent re-settings of the experiment for the detection of turning-point or subcritical bifurcations. The approach exemplified here addresses these issues drawing from ideas of numerical bifurcation detection. The idea is to couple the experiment with an on-line computer-assisted identification/feedback protocol so that the closed-loop system will converge to the open-loop bifurcation points. We demonstrate the applicability of this instability detection
\end{abstract}

\footnotetext{
*Corresponding author. E-mail: ramiro@arnold.princeton.edu, Telephone +5246117802, Fax +52-46117744
} 
method by real-time, computer-assisted detection of period doubling bifurcations of an electronic circuit; the circuit implements an analog realization of the Rössler system. The method succeeds in locating the bifurcation points even in the presence of modest experimental uncertainties, noise and limited resolution. The results presented here include bifurcation detection experiments that rely on measurements of a single state variable and delay-based phase space reconstruction, as well as an example of tracing entire segments of a codimension-1 bifurcation boundary in two parameter space.

PACS numbers: 05.45.,82.40

Keywords: Bifurcation Detection, Nonlinear Systems, Adaptive Control. 


\section{Introduction}

Over the last 30 years increasing efforts have been directed towards the understanding of experimentally observed complex dynamical behavior. The development of Nonlinear Dynamics as a branch of applied mathematics provides us the set of tools necessary for understanding the response of nonlinear dynamical systems as operating parameters are varied. Characterization of the nonlinear dynamical behavior of experimental systems, however, remains a difficult task. This is mainly because real systems cannot be manipulated as easily or as cleanly as a model would on the computer when its bifurcation behavior is traced.

Typically, an experimental bifurcation diagram is obtained via repeated application of a set-and-observe procedure. The operating parameter of interest is set and the system dynamics allowed to evolve asymptotically to stationary (e.g. steady or oscillatory) behavior. The operating parameter setting is then changed (e.g. incremented slightly) and the experiment is repeated; if a qualitative difference is detected between the asymptotic dynamics reached for two consecutive parameter settings, a bifurcation is inferred to have occurred somewhere in-between. A similar approach can be applied to the computational detection of bifurcations if one is only allowed to use forward-in-time integration of the model equations (i.e. set parameter values and initial conditions and run a simulation to stationarity). In both cases there are serious drawbacks. For example, in the investigation of a period doubling bifurcation of a periodic orbit, as the critical value of the search parameter is approached the dynamics will slow down considerably, and the time required to determine whether ultimately single loop or double loop oscillations are observed increases dramatically. Furthermore, if one wishes to detect a subcritical bifurcation, or a saddle-node bifurcation, once one steps over the critical parameter value, the system will move far away from the phase space region of interest. The experiment will then need to be reset to reach the vicinity of the stable solution located just before the critical parameter value that was overstepped.

In previous work [1], 2] we proposed a protocol for the experimental detection of bifurcations motivated by the procedure of computational construction of bifurcation diagrams from mathematical models. Numerical bifurcation theory in a computational environment allows the bifurcation location via a simultaneous search for criticality in (phase) $\times$ (parameter) space. An 
augmented set of equations (e.g. steady state equations plus a criticality condition) is typically formed, and its fixed point (steady state values at criticality and critical parameter value) is converged upon quadratically using a Newton-Raphson contraction mapping for the augmented system of equations. Obviously in this case the states do not evolve on the computer based on the system dynamics, but based on the computational contraction mapping dynamics.

The proposed protocol for the detection of bifurcations seeks an "experimentalcomputational" compromise: an experiment (i.e. states evolving based on system dynamics) is coupled to a parameter evolution law which is based on criticality conditions and feedback control. The augmented, closed loop system, constructed in this manner has a stable steady state at what used to be the open loop system's marginally stable steady state. The computational building blocks that constitute the parameter evolution law are based on the on-line identification of a "local nonlinear" dynamic model, obtained via least-squares regression [10], used to estimate criticality on-line, and simple feedback control algorithms that use the bifurcation parameter as the actuator in order to drive the system towards the bifurcation point. As it will be illustrated below, this algorithm enhances our ability to efficiently detect instabilities, trace segments of the bifurcation diagram ("operating diagram"), and, in general, characterize the system dynamics in multiparameter space. The "experimental" system used in our illustration is an analog realization of the Rössler system. For this system, we converge experimentally on the period doubling bifurcation of simple periodic orbits in one and two-parameters. The local adaptive model used to infer the presence of the bifurcation is a 2D nonlinear map. Our illustrations are based on (a) full state vector measurement; and (b) reconstruction of the state-space based on time series of a single state measurement and the use of delay coordinates.

An alternative motivation for studying this type of problem is the typical Ziegler-Nichols (ZN) tuning procedure for PID (proportional- integralderivative) controllers [16], which motivated the work of O'Neil, Lyberatos and Svoronos [9] on adaptive determination of bifurcation points. In the "textbook" ZN procedure the "experiment" is the closed loop system with a proportional controller; the gain of this controller is the "bifurcation parameter". The closed-loop system loses stability when the proportional gain $K$ reaches the value $K_{u}$. This critical parameter value is precisely the ZN "ultimate gain". In practice, one obtains $K_{u}$ by slowly increasing the gain of the 


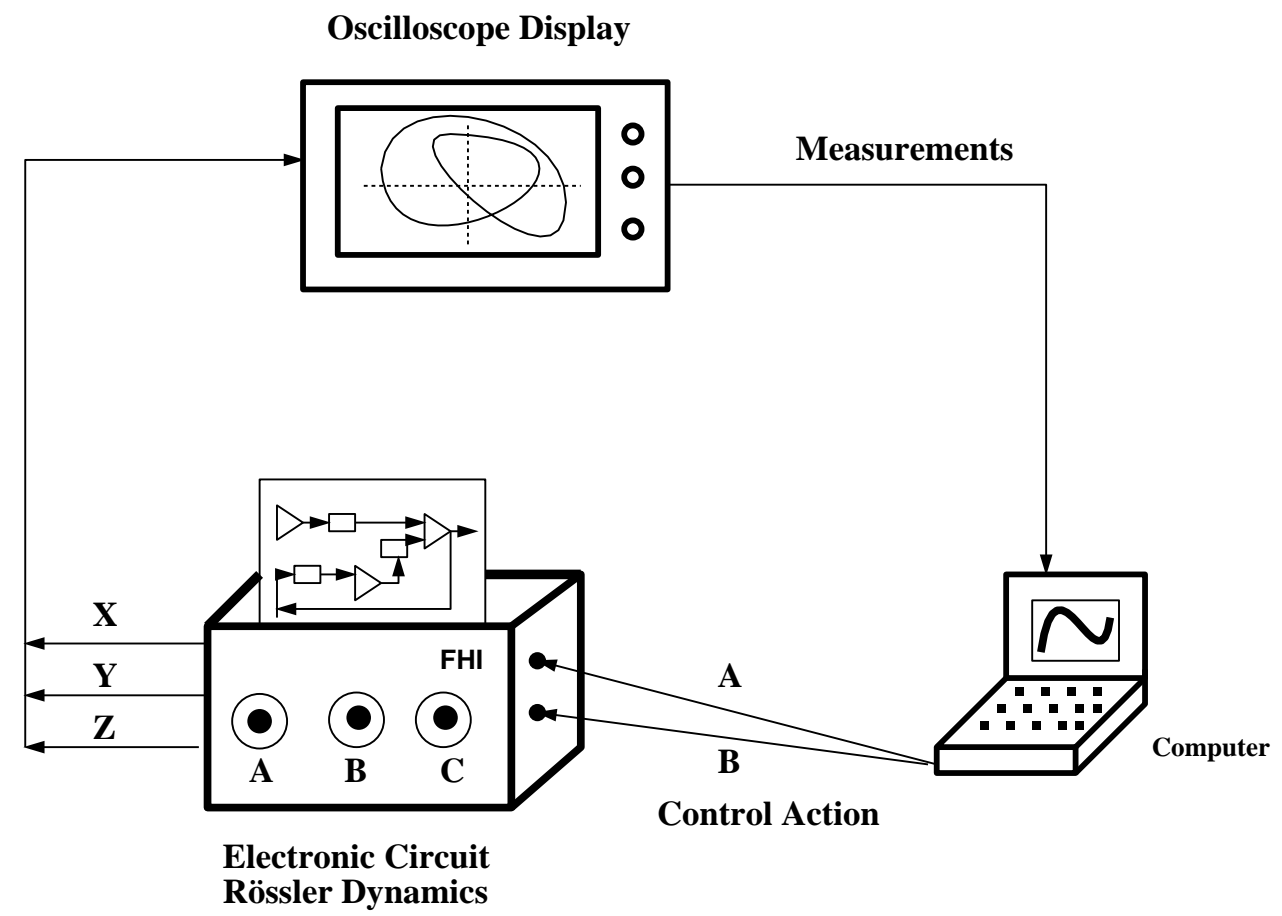

Figure 1: Schematic of the experimental setup.

proportional controller until one observes incipient oscillations. Knowledge of the location of the bifurcation and the frequency of the oscillations dictates then the ZN-tuning settings for a PID controller; it is interesting that this practical controller tuning approach is based on experimental bifurcation detection.

The paper is organized as follows: first, we describe our system (the electronic Rössler circuit) and the bifurcation detection protocol before presenting our experimental results. We then provide some concluding remarks.

\section{The Rössler Circuit}

The Rössler model was proposed in the early 1970's [13 as one of "the simplest" nonlinear vectorfields capable of generating chaotic behavior. It consists of the following set of three ordinary differential equations: 


$$
\begin{aligned}
& \frac{d x}{d t}=-y-x \\
& \frac{d y}{d t}=x+a y \\
& \frac{d z}{d t}=b+z(x-c)
\end{aligned}
$$

This system has been extensively studied. For $b=2$ and $c=4$ and as $a$ is increased, it exhibits a steady state undergoing a Hopf bifurcation followed by a cascade of period doubling bifurcations of the periodic orbits culminating in an apparently chaotic attractor around $a=0.396$.

In the implementation of the protocol for the adaptive detection of experimental bifurcations, we use as model system an analog realization of the Rössler system. The Rössler equations are implemented via a sequence of feedback loops of electronic signals. The circuit was manufactured at the FHI labs" and includes an encasing that allows both manual and digital manipulation of all three system parameters. In our experiments the parameter dynamics were computer-generated in closed loop, and were communicated to the device through its digital I/O channels. The circuit is coupled (Fig. 1) with a VMEbus System (32bit backplane system) consisting of a VMEbus CPU-board Baja40 from Heurikon Division controlled via a Motorola 68040 32-bit microprocessor chip running internally with $66 \mathrm{MHz}$, externally with $33 \mathrm{MHz}, 32$ MByte RAM and several I/O interfaces and a VMEbus IP carrier board with four IndustryPack slots. One slot was used with a TIP501 optically isolated 16 channel 16 bit ADC module from TEWS Datentechnik, input voltage +/- 10 volt, 14.5 usec data acquisition and conversion time, accuracy +/- 4 LSB, linearity +/- 4 LSB, and the other slot with a TIP550 optically isolated 4 channel 12 bit DAC from TEWS Datentechnik, output voltage +/- 10 volt, 13 usec settling time, accuracy +/- 1 LSB, linearity +/1 LSB. The array uses an operating system VxWorks 5.3.1 (Tornado) from Wind River System. The data acquisition components allow us to specify the accuracy of the measurements from 11 to 16 bits.

The dynamic response of the motivating set of differential equations is well reproduced. Figure 2 presents a comparison of a couple of projections

${ }^{1}$ A detailed diagram of the circuit is available in the web in the following address http://w3.rz-berlin.mpg.de/pc/spatdyn/spatdyn.cgi?Publications 
of the double-loop limit cycle predicted and observed at $a=0.35, b=2$ and $c=4$. The deviation of the signal given by the circuit with respect to the one obtained by integrating the differential equations is minimal, both in the actual values of the variables as well as in the period of the oscillations. Similar results are observed within the range of operation of the circuit: $a \in[0,1], b \in[0,10], c \in[0,10]$ and values of the state variables inside the range $[-10,10]$.

\section{The Bifurcation Detection Protocol}

The building blocks that constitute the bifurcation detection protocol are schematically depicted in Figure 3. They involve four different modules: one that identifies on-line an approximate model of the system, a second one that uses the identified model to estimate the location of the bifurcation point in phase $\times$ parameter space; this second module relies in the information provided by a test function (third module) that contains the conditions defining the type of bifurcation sought. The fourth and final module uses the estimated critical parameter value (our estimate of the open-loop critical conditions) to devise a policy that will bring and keep the system closer to open-loop criticality. The repeated application of the algorithm, composed by these four modules, drives the system to the true open-loop bifurcation point, and holds it there. In devising the algorithm, we assume that at least one of the states of the experimental system can be observed (measured), and that at least one operating parameter is available to be manipulated (the bifurcation will be located with respect to this parameter). Furthermore, we assume that the open-loop system is in the neighborhood of a local bifurcation; thus its dynamics can be captured via a low-order nonlinear model. Using such a low-order approximation to model the dynamics of the system is motivated (and can be justified) by the existence of a normal form for the bifurcation. The discrete-time model used in the system identification module of the algorithm ((1) in Fig. 3) takes the form of a low order polynomial on the observed variables and the parameter, motivated from Taylor series

expansions in the neighborhood of the bifurcation point, and the theory of Center Manifolds/Normal Forms. The polynomial model is of the type:

$x_{i}(k+1) \equiv F_{i}[\mathbf{x}(k) ; p(k)]$ 


$$
\begin{aligned}
= & a_{i}+\sum_{j=1}^{n} b_{i, j} x_{j}(k)+c_{i} p(k)+\sum_{j=1}^{n} \sum_{l=1}^{n} d_{i, j, l} x_{j}(k) x_{l}(k) \\
& +\sum_{j=1}^{n} e_{i, j} x_{j}(k) p(k)+\sum_{j=1}^{n} \sum_{l=1}^{n} \sum_{m=1}^{n} f_{i, j, l, m} x_{j}(k) x_{l}(k) x_{m}(k) \quad(i=1, \cdots, n)
\end{aligned}
$$

where $\mathbf{x}$ represents the $n$-dimensional vector of state variables, $p$ is the parameter, $a, b, c, d, e$ and $f$ are the polynomial coefficients, and $k$ is the iteration counter. Note that the polynomial is linear in the parameter and contains cubic terms on the state variables. The normal forms of the most common bifurcations are linear in the parameter, and the normal form of the Hopf bifurcation has cubic terms in the state variables [15]. The polynomial can be simpler for certain specific types of bifurcation; the form presented here is appropriate for the detection of local codimension-1 bifurcations. For the experimental system, we rely in Poincaré sections and/or reconstructions based on delayed measurements to form the vector of states. The identification module uses a least-squares algorithm [10] to fit the coefficients of the polynomial from experimental observations; other algorithms, such as the projection method, can of course also be used. The model is updated at every step as new observations become available. In order to prevent illconditioning of the least-squares algorithm during the experimental run, a well-known problem in the on-line identification of experimental systems, the parameter signal must be persistently excited (here, by addition of random noise) [3, 0].

The second module of our algorithm ((2) in Fig. 3) uses the polynomial model identified by the first module to estimate the location of the open-loop bifurcation. This is a computational step that involves the simultaneous solution of the steady state equations along with a criticality condition appropriate for the bifurcation sought ((3) in Fig. 3). This is accomplished using an algebraic equation solver (usually a contraction mapping like a NewtonRaphson). For example, for a period-doubling bifurcation, which we detected in the Rössler circuit, the bifurcation is located at the point where one Floquet multiplier (an eigenvalue of the linearization around the fixed point of the open-loop Poincaré map model) is equal to - 1 . Thus, a suitable test function, for a two-variable discrete-time system, will be $\operatorname{det}[\mathbf{I}+\mathbf{J}]=0$, where $\mathbf{I}$ is the identity matrix and $\mathbf{J}=\left[\nabla_{x} \mathbf{F}[\mathbf{x} ; p]\right]$ is the Jacobian of the identified model at the steady state $(\mathbf{x}, p)$.

In order to find the location of the critical state $\left(\left\{\hat{\mathbf{x}}_{c r}, \hat{p}_{c r}\right\}\right)$, one must 
solve for $\{\mathbf{x}, \mathbf{p}\}$ such that (period doubling case):

$$
\begin{aligned}
x_{i}-F_{i}[\mathbf{x} ; p] & =0 \quad(i=1, \cdots, n) \\
\operatorname{det}[\mathbf{I}+\mathbf{J}] & =0
\end{aligned}
$$

The final module ((4) in Fig. 3) uses the identified model and the estimated location of the bifurcation $\left(\left\{\hat{\mathbf{x}}_{c r}, \hat{p}_{c r}\right\}\right)$ to find a parameter variation protocol that will drive the system to the bifurcation point. This critical step can be accomplished using several alternative "feedback laws". Previously [1, 2], we had illustrated the use of one of the simplest: a policy that will bring the system to criticality in the minimal possible number of steps (dead-beat policy). In the runs presented below, however, we rely on "softer" policies in order to overcome problems associated with the local character of the model identified, the limited resolution of measurements and the need for continuous excitation of the control signal associated with the ill-conditioning of the least squares identification procedure. In addition, the control policy should be possible to be evaluated and implemented very fast (almost instantaneously) in real time. This is because we have ignored in our experiment the delays associated with I/O tasks as well as those associated with the computation of the control action - we have effectively assumed that computation and implementation of control action is instantaneous. This avoids a number of complications and possible instabilities having to do with physical delays in the control loop. These are important issues, and will be taken up in future work; they are, however, more "implementation difficulties", and do not directly pertain to the mathematical underpinnings of our procedure.

With these restrictions in mind, we explored a couple of plausible two parameter variation protocols: the first policy attempts to minimize the distance from the critical point in the next iteration, penalizing large parameter variations; the second protocol is based on decreasing the distance to the critical state (in phase space) in the next iteration by a fixed fraction. In the notation used above, the first protocol results from:

$$
\min _{p(k)} \sum_{i=1}^{n}\left(x_{i}(k+1)-\hat{x}_{i, c r}\right)^{2}+W\left(p(k)-\hat{p}_{c r}\right)^{2}
$$

Note that, since the identified model (used to predict $\mathbf{x}(k+1)$ ) is linear in the parameter $p$, the solution of this minimization problem renders a linear 
equation for $p$. The parameter $W$ is a penalty weight, which we usually set in the range $[0.1,10]$. Since the model form used is only valid locally, close to the open-loop bifurcation, its predictions away from the bifurcation are not accurate. In the presence of modeling errors, a dead-beat policy may drive the system farther away from the bifurcation instead of bringing it closer. The introduction of the weight $W$ gives us a mechanism to moderate excessive control action, and thus, to possibly overcome the effect of such modeling errors away from the bifurcation point. A more appropriate implementation of this policy would seek only to penalize actions that bring the system outside the range of validity of the model. That is, the penalty should only be applied if the prescribed control action is larger that the estimated range of validity of the identified model. We are currently exploring this issue.

The second policy is motivated by the form of transients approaching a steady-state near a Hopf bifurcation. In such a scenario the dynamical system will slowly converge to a marginally stable steady state by (possibly fast, but slowly decaying) spiraling towards it. Thus, this second policy attempts to control the speed of the approach by requiring that the distance from the critical state location in the next iteration becomes a fraction of the current distance from criticality. In mathematical terms we solve for $p(k)$ such that:

$$
\sum_{i=1}^{n}\left(x_{i}(k+1)-\hat{x}_{i, c r}\right)^{2}=C^{2} \sum_{i=1}^{n}\left(x_{i}(k)-\hat{x}_{i, c r}\right)^{2}
$$

Here $C$ is the desired fractional reduction, in the next iteration, of the distance from estimated open-loop criticality. We typically set $C$ in the range $[0.9,0.95]$. Since the model to predict $x_{i}(k+1)$ is linear in $p$, the solutions of this equation are the roots of a quadratic equation that can be solved explicitly. The root selected is the one closer to the current value of the parameter.

Both control policies must be applied repeatedly in order to drive the closed loop system to the point that, for the open-loop system, was a marginally stable solution. Although the number of iterations to reach the bifurcation may be large (e.g. more than 100 iterations), we have found that, even with these simple policies, the closed-loop system performs well, finding the bifurcation for every experiment independently of the initial condition used.

By using the identification and control methods in the manner described, we circumvent the long settling periods associated with open-loop near- 
critical dynamics. As previously illustrated in [2] through computational modeling, this methodology is also capable of driving the system back to steady state after (mildly) overshooting the critical value of the bifurcation parameter even for "hard" bifurcations such as turning points or subcritical Hopf bifurcations. In the following section, we illustrate our approach by locating period-doubling bifurcations of periodic orbits for the Rössler circuit device.

\section{Experimental Runs.}

A period-doubling cascade of periodic orbits (or fixed points of maps or Poincaré maps) is perhaps one of the best understood routes to deterministic chaos. The Rössler system exhibits this type of transitions at $b=2$ and $c=4$ for increasing values of the remaining parameter $a$. The first of such period doubling bifurcations is observed for $a \approx 0.33485$. It is precisely this bifurcation that we set to locate using the protocol described above.

Poincaré sections provide a natural framework for observing/reporting period doubling of periodic orbits. The construction of our approximate models is performed on such a (transversally intersecting the flow) Poincaré section. Initially, we measure all three state variables from the circuit $(x, y, z)$ and define the Poincaré section as the plane $x=0.2$, crossed towards increasing values of $x$. For this Poincaré section the critical point is located at $y=-4.1409$ and $z=0.44601$. In the proposed protocol, the model to be identified involves two state variables (the coordinates $(y, z)$ on the Poincaré section) and one parameter $(a)$. Thus, our model will try to predict where the trajectory intersects the plane at the next iteration $\left(y_{k+1}, z_{k+1}\right)$ based on the current location $\left(y_{k}, z_{k}\right)$ and the value of the parameter $\left(a_{k}=p_{k}\right)$. For the experiment, the intersection point on the surface defining the Poincaré surface was obtained via fast (between 200 to $1000 \mathrm{~Hz}$ ) sampling of all variables and linear interpolation between the sampled points once the crossing with the surface was detected.

For the circuit, the period of the oscillations before the bifurcation is approximately $0.62 \mathrm{~s}$. In comparison with the Rössler ODE model, the arbitrary units of time translate as seconds for the circuit device and the arbitrary units of the state variables and parameters as voltages measured in Volts. Using a 16-bit data acquisition card, the measurement resolution for 
the state variables is approximately 0.0006 volts and 0.00003 volts for the parameter $a$ which has a smaller range of variation (voltage). As we mentioned earlier, in order to successfully identify a varying on-line model, one should apply persistent excitation to the actuator (the parameter signal). This is done in the form of random noise with maximum amplitude of 0.003 volts. The excitation signal prevents the system from reaching stationary states for which the measurement will be effectively constant. Such measurements will render the matrix used in the identification computations severely illconditioned, and the approximate model will deteriorate. With persistent excitation signals the system will be always "on the move" allowing the collection of information-rich data resulting in more accurate models. Since, however the final goal is to drive the system to the open-loop bifurcation, very large excitation signals are undesirable because they work against the control actions implemented. The excitation level selected represents a compromise between these competing effects. Depending on the system under consideration, the magnitude of the excitation signal should be adjusted. In our experience with several different simulated systems, we have found that the amplitude of the excitation signal must be at least one order of magnitude larger than the resolution available in the measurement.

The use of this relatively large excitation signal combined with the soft control policies described previously constrains the selection of the appropriate convergence criterion. The approach to the critical state may be slow, possibly also "erratic" due to the effect of the excitation; on the other hand, the system may become trapped and the limited resolution may translate in biased models and incorrect criticality predictions.

In order to prevent the latter occurrence, we opted for a two-level convergence criterion: (1) we require that the norm of the deviation from criticality in the extended phase $\times$ parameter space is sufficiently small (i.e. less than 0.008 volts) for a relatively small number of consecutive iterations (typically $5)$; and (2) after complying with the first criterion, we restart the system identification procedure by gathering enough data to construct a "fresh" model, using the excitation signal without control, and then restart the feedback control until the first criterion is again satisfied. Overall convergence is declared only if two consecutive restarts reach the same point within the tolerance indicated above. The first criterion does not excessively burden the controller performance, while the second seeks to prevent declaring convergence at a possibly unconverged, incorrect prediction of the location of the 
bifurcation. The tolerance to declare convergence (0.008 volts in our case) should clearly be larger than the set maximum amplitude of the random noise (excitation signal, 0.003 volts); a reasonable rule-of-thumb is to set this tolerance half-an-order of magnitude above the maximum amplitude of the random noise. We note, however, that the achievable accuracy in locating the bifurcation is limited, not because of the convergence criterion, but most importantly because of the excitation signal, model sensitivity and, ultimately, limited resolution in the measurements.

With the feedback protocols implemented, we observed, during our runs, that large parameter variations ("control actions") arose frequently. Since such variations may drive the system outside the range physically accessible to the circuit device (i.e. negative parameter values), or outside the range for which sustained oscillations are observed (thus, preventing the trajectories from intersecting the plane used in the definition of the Poincaré section) we opted for establishing hard bounds ("saturation") for the values that the parameter can take. If the control action prescribed goes beyond these bounds, the parameter is set to the value of the bound that has been surpassed (the actuator saturates). For the run at $b=2$ and $c=4$, the minimum value allowed for $a$ was 0.12 and the maximum 0.5. Below $a \approx 0.125$ the only attractor of the system is a steady state, while above 0.5 the chaotic attractor observed in the range $[0.396,0.5]$ disappears via global bifurcations and the state variables of the circuit tend to saturate.

With the hardware available to us, the evaluation of the protocol, including identification of an updated model, location of criticality and evaluation of the control policy, involved between 30 and 60 milliseconds, depending on the number of evaluations required for some of the subroutines such as the algebraic solver to find criticality. The effect of such "large" delay (about one tenth of the period of oscillation in the worst case) was often detrimental to the performance of the protocol, preventing the accurate location of the bifurcation. In order to overcome this situation, we modified the evaluation order of the subroutines. That is, we first calculate and implement a control policy using one-iteration-old models, then we use the collected data to update the model and prediction of criticality for the next iteration. This strategy was successful, since the evaluation of the control policies proposed require only the solution of linear or quadratic equations and thus take little time (less than $3 \mathrm{~ms}$ ), and the slow approach to the critical state makes consecutive models very similar most of the time (depending on the conditioning 
of the matrix involved in the identification of the model).

Figure $⿴$ illustrates the performance of the protocol. The parameter was initially set at $a=0.42$. At this parameter setting, the open-loop circuit exhibits a chaotic attractor. The experiment is left to evolve in the vicinity of this parameter value for about 30 iterations, while data are gathered to construct an initial model. After approximately 70 iterations, the system has been driven to the vicinity of the bifurcation. The last 50 iterations, for which the response of the circuit is practically constant, represents the period needed to declare convergence with one restart as described above. For this run the critical state was predicted at $y=-4.1127, z=0.4537$ and $a=0.3389$. This compares favorably with the location of the bifurcation for the Rössler ODE system. As we indicated before, we anticipated that the estimation of the bifurcation location will not be very accurate because of the persistent excitation signal and soft control policies. A more representative result should be reported after gathering statistics over a number of runs. On average, over 15 runs, each run with different initial condition and initial parameter value, the bifurcation is predicted to be located at $a=0.3309$ with a standard deviation of 0.0082 volts, with the critical state located at $y=-4.0964$ and $z=0.4552$ and standard deviations of 0.0134 and 0.0014 volts respectively. The deviation of this average is well within the range allowed for declaring convergence in our runs and the standard deviation commensurate with the excitation signal and convergence criteria. We note that all our runs did converge to the critical state albeit occasionally at a larger number of iterations than for Figure 4 .

Figure 5 presents another run that illustrates the role of hard, saturation bounds limiting the values that the parameter can take during the search for criticality. After approximately 40 steps, an incorrect criticality prediction translates into a drastic control action. Since the value prescribed for the parameter would be below the preset lower bound, the parameter is set to the bound. The performance of the controller deteriorates and the system is trapped near the lower bound for about 40 iterations before restarting its movement towards the critical state.

Figure 6 shows an example of the phenomenon commonly referred to as "bursting". The system starts its gradual approach toward criticality, but since the coordinates of the points in the trajectory do not change much, the matrix used for the identification becomes progressively more ill-conditioned, eventually rendering a "bad" model that, in turn, dictates a large parameter 
excursion ("burst"). The trajectory to criticality exhibits, in this example, a couple of instances of such bursting on its route to the eventual correct detection of the critical fixed point.

For those runs initialized at parameter values lower than the bifurcation value, as the one illustrated in Figure 6, the convergence to the critical point was consistently declared for slightly lower values of the parameter than the true critical parameter value. In contrast, when the initialization took place at conditions for which the open loop system exhibited apparently chaotic oscillations (i.e. Figure 1 ), the collection of information-rich data allowed the accurate estimation of the critical fixed point location early on, resulting in faster convergence towards the bifurcation.

One potential limitation of the application of this strategy may be the available measurement resolution. The examples presented above rely on a 16 bit A/D card. However, as illustrated in Figure 7, the bifurcation was successfully located also for lower resolution, equivalent to that provided by a standard 11 bit A/D converter (i.e. one order of magnitude less in resolution for the parameter and state variables).

Although the examples discussed until this point involve measurements of all the states of the system, it is also possible to use only a single measurement time series. Figure 8 illustrates the performance of the protocol for the location of the same bifurcation using only measurements of the variable $x$ and relying on delayed measurements to reconstruct the phase space. The delay $(\tau)$ for the reconstruction was set to $40 \mathrm{~ms}$. In practice one could seek to find an optimal value of the delay using, for example, mutual information measures; this was not done here. The Poincaré section was set as before using the plane $x(t)=0.2$. The critical state is located for the Rössler system at $x(t-\tau)=-1.1701$ and $x(t-2 \tau)=-2.2020$. On average over 5 runs, the bifurcation is predicted at $a=0.3235$ for $x(t-\tau)=-1.1143$ and $x(t-2 \tau)=-2.1320$, with standard deviations of $0.0080,0.0042$ and 0.0010 volts respectively. The trajectory to reach the critical state, however, exhibits a larger number of instances of bursting and saturation of the parameter and thus requires a larger number of iterations as illustrated in Figure 8 . This deterioration in performance of the protocol can be attributed to the increased sensitivity of the identification resulting from the use of a suboptimal delay and the smaller effective resolution of the sampled variable.

A final test of the capabilities of the proposed protocol for the detection of bifurcations comes by attempting to draw, experimentally, a two-parameter 
bifurcation diagram. That is, we attempt to trace automatically a segment of our codimension-1 bifurcation boundary in two parameters. We vary the (second) parameter $b$ in the range $[1,3]$. Starting at $b=3.0$, we locate the value of $a$ for which the bifurcation is observed and then decrease $b$ by a fixed amount (0.05 volts). Figure 9 presents the comparison of these runs for our circuit device, and the computed boundary for the Rössler ODE system. The results presented are the average over five sweeps, three calculated decreasing $b$ and two increasing it. The previously found bifurcation point was used as the starting point for the new search with a different value of $b$. Figure 10 presents the comparison of the location of the (open loop critical) fixed points for the Poincaré section. The boundaries for the Rössler ODE system were calculated using AUTO [5]. As can be seen in Figures 9 and 10 the segment is accurately located, as are the critical states. For both plots, standard deviations have been omitted; they are comparable to the previously indicated values for the single parameter runs.

\section{Conclusions}

We have presented a successful implementation of an algorithm for efficiently driving an experimental system to (what at open loop would have been) criticality. The control protocol was applied to an experiment in the form of an electronic circuit device that implemented the dynamics of the Rössler system. The proposed algorithm is based on adaptive system identification, separation of time scales close to criticality, and on the exploitation of the identified local model to search for the criticality conditions; the estimation of open-loop criticality is used to devise a control policy that drives the closed-loop system towards the bifurcation. The protocol seeks, in a sense, to mimic the procedure used in the numerical construction of bifurcations from mathematical models: the critical state of the open-loop system becomes a stable steady state of the augmented system in (phase) $\times$ (operating parameter) space. We illustrated the capabilities of the algorithm in the location of period doubling bifurcations of periodic orbits. The bifurcation was successfully located even in the presence of limited resolution. We also illustrated the applicability of this approach when only one state variable can be measured by reconstructing the phase space using delayed measurements. Our final illustration involved the tracing of a segment of the two parameter 
bifurcation boundary.

Our selection of an electronic circuit device with Rössler dynamics, to demonstrate the experimental feasibility of the protocol for the automatic detection of bifurcations, was intended to provide a testing ground for assessing the performance of the protocol. The circuit device provides us with a highly reproducible and easily manipulated experiment with a well charted dynamical behavior. We chose the period doubling bifurcations of the oscillations of the circuit as our representative codimension-1 bifurcation. The low-dimensionality of the model used crucially depends on the time-scale gap between the long time scale associated with the critical eigenvalue(s) and the time scale of the "next least stable" eigenvalue. It is important that all operations performed in our system (I/O as well as software computations) are fast compared to this "next least slow" time scale. Since the return time for our Poincaré map was roughly $600 \mathrm{~ms}$, our relatively short (approximately $40 \mathrm{~ms}$ ) action delay could be considered "approximately instantaneous". An alternative that would speed up the protocol evaluation is the use of recursive least squares techniques [3, [7] that may reduce significantly the time involved in the identification of a local model; however, this alternative resulted in more sensitive and prone-to-burst trajectories, even though we attempted several different strategies in the assignment of forgetting factors for the identification. Other alternatives, such as the use of projection algorithms [7], are also feasible, but were not investigated.

Perhaps the most limiting aspect for the widespread application of the proposed protocol involves the high sensitivity of the strategy as a result of the combination of the control policies, excitation signal and on-line identification of a model with only local validity. Such sensitivity translates in erratic and slow motion towards the bifurcation, frequent bursts, trapping of trajectories near hard bounds and ill-conditioning of the identification process.

We are currently working on posing the entire problem (system, identification and control) as an augmented dynamical system, for which the existence of an overall converged state can be demonstrated, and its stability quantified. Such an approach could also allow us to explore issues related to the performance in the presence of multiple nearby bifurcations of the same type.

Extensions of the algorithm are straightforward: higher codimension bifurcations can be detected by solving the corresponding (more than one) 
criticality conditions for the (open-loop) marginally stable steady state and the (more than one) critical parameters. In principle one might even be able to accommodate unstable, nonbifurcating parts of the spectrum on the right-hand plane. Several important elements can be improved upon: (a) Formulation of the criticality conditions in a less naive and computationally more efficient manner, along lines of modern numerical bifurcation theory (e.g. [5] and references therein); (b) Usage of more sophisticated (e.g. receding horizon) feedback control laws [6]; and (c) Improvement of the type of local model used. While in this problem we used polynomial models based on Taylor-series expansions and normal forms, it is obvious that other types of nonlinear dynamic models (e.g. models based on artificial neural networks) can also be used in this context; we, among many others, have demonstrated that such models are capable of accurately predicting the bifurcation structure of a nonlinear system when trained with time series data [11, [12].

While we are continuing theoretical and computational work along the directions described above, we are also implementing this approach in the study and characterization of the bifurcations of electrochemical systems [8]

It may be interesting to note that this approach can also be coupled with large scale scientific simulators, for which the relevant long term dynamics are low-dimensional, in order to chart their bifurcation scenario [4] (as opposed to writing a bifurcation algorithm for them from scratch). The strategy of seeking and stabilizing a fixed point with one additional condition (here, open-loop criticality), falls in the same category as extremum seeking controllers [14]; controller developments along these lines would find direct application in our protocols, and vice versa. We believe that an approach like the one described here can eventually become a standard real-time tool which will assist experimentalists in efficiently detecting bifurcations and tracing operating diagrams; such a tool could drastically enhance the experimental characterization of nonlinear dynamics in multiparameter space.

\section{Acknowledgements}

We are grateful to Professor Ertl for his interest in and generous support of this work. We thank Heinz Junkes and Georg Heyne from the FHI tech-

nical staff for technical support. We gratefully acknowledge the support of CONACYT and COSNET (RRM), NATO (IGK, KK), and AFOSR (IGK) 
for partial funding of this research. IGK and RRM gratefully acknowledge the support from the Alexander von Humboldt Foundation.

\section{References}

[1] J. S. Anderson, I.G. Kevrekidis, R. Rico-Martínez and K. Krischer, Use of adaptation and feedback in the experimental detection of bifurcations, Proc. 30th Annual Conf. on Information Sciences and Systems, 1:155160, 1996.

[2] J. S. Anderson, S. Y. Shvartsman, G. Flätgen, I. G. Kevrekidis, R. RicoMartínez and K. Krischer, An adaptive method for the experimental detection of instabilities, Physical Review Letters, 82:532-535, 1999.

[3] K.J. Aström and B. Wittenmark, Adaptive Control, Addison-Wesley, New York, NY, 1989.

[4] G. S. Copeland, I.G. Kevrekidis and R. Rico-Martínez, Adaptive detection of instabilities and nonlinear analysis of a reduced-order model for flutter and rotating stall in turbomachinery, Proceedings of the IEEE International Conference of Control Applications (CCA, Hawaii), Paper TuP3-1, 1146-1150, 1999.

[5] E. Doedel, H.B. Keller, and J.P. Kernévez, Numerical analysis and control of bifurcation problems: (I) Bifurcation in finite dimensions, Int. J. of Bifurcation and Chaos, 1:493-520, 1991.

[6] J. W. Eaton and J. B. Rawlings, Feedback control of nonlinear processes using on-line optimization techniques, Computers chem. Engng, 14:469479, 1990.

[7] G.C. Goodwin and K.S. Sin, Adaptive Filtering, Prediction, and Control, Prentice-Hall, Englewood Cliffs, NJ, 1984.

[8] K. Krischer, Principles of temporal and spatial pattern formation in electrochemical systems, Mod. Aspects Electrochem., 32:1-142, 1999. 
[9] D.G. O'Neil, G. Lyberatos, and S.A. Svoronos, Adaptive Determination of Bifurcation Points, Presentation at AIChE Annual Meeting, Paper 172j, Washington, D.C., 1988.

[10] W.H. Press, S.A. Teukolsky, W.T. Vetterling, and B.P. Flannery, Numerical Recipes in C, 2nd ed., Cambridge University Press, New York, NY, 1992.

[11] R. Rico-Martínez, J.S. Anderson and I.G. Kevrekidis, Continuous-time nonlinear signal processing: A neural network based approach for gray box identification, Proc. 1994 IEEE Workshop on Neural Networks for Signal Processing, 596-605, 1994.

[12] R. Rico-Martínez, I.G. Kevrekidis and K. Krischer, Nonlinear system identification using neural networks: dynamics and instabilities, Chapter 16 in the book Neural Networks for Chemical Engineers, A.B. Bulsari (ed.), Elsevier Science B.V., 1995.

[13] O. E. Rössler, An equation for continuous chaos, Phys. Lett., 57A:397398, 1976.

[14] H-H Wang, S. Yeung and M. Krstic, Experimental application of extremum seeking on an axial flow compressor, IEEE Trans. on Control System Technology, 8:300-309, 2000.

[15] S. Wiggins, Introduction to Applied Nonlinear Dynamical Systems and Chaos, Springer-Verlag, New York, NY, 1990.

[16] J. G. Ziegler and N. B. Nichols, Optimum Settings for Automatic Controllers. Trans. ASME, 64:759, 1942. 

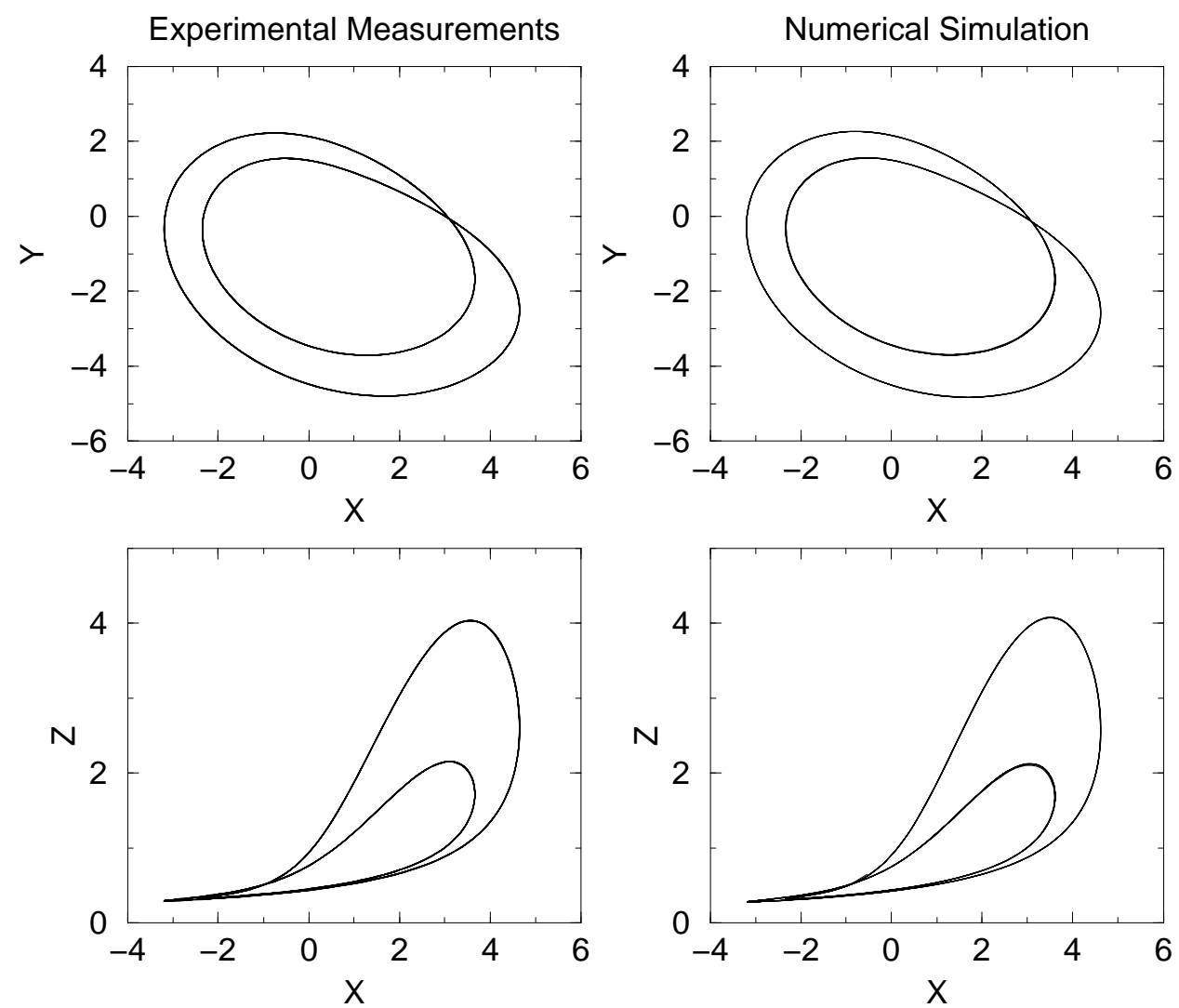

Figure 2: Comparison of attractors for the circuit (left) and the numerically computed trajectory obtained integrating the set of ODEs of the Rössler system. The comparison is made for a double-loop trajectory observed at $a=0.35, b=2$ and $c=4$. 


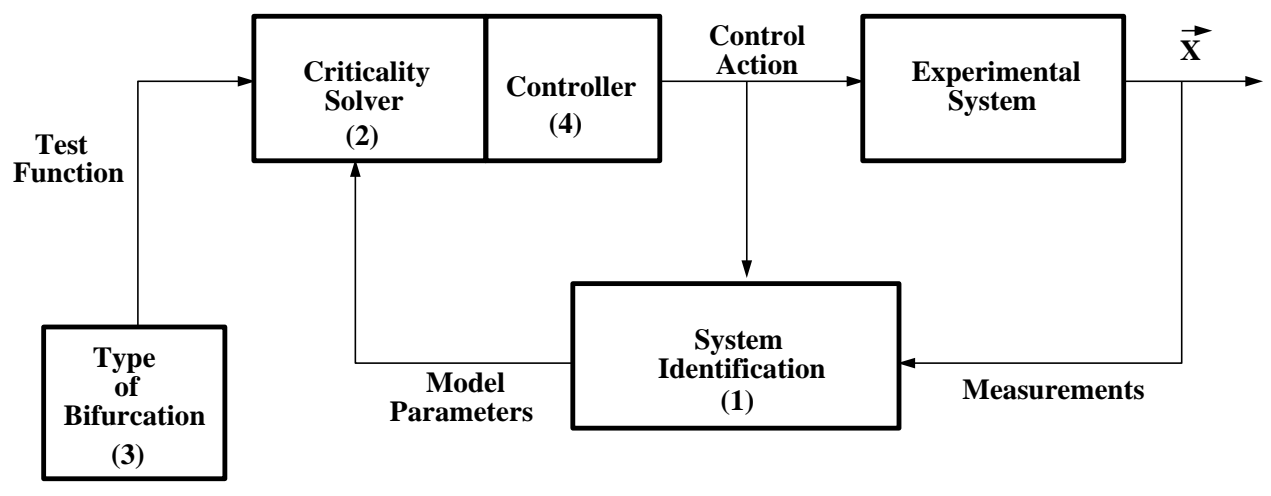

Figure 3: Schematic diagram of the algorithm for the experimental detection of bifurcations. 

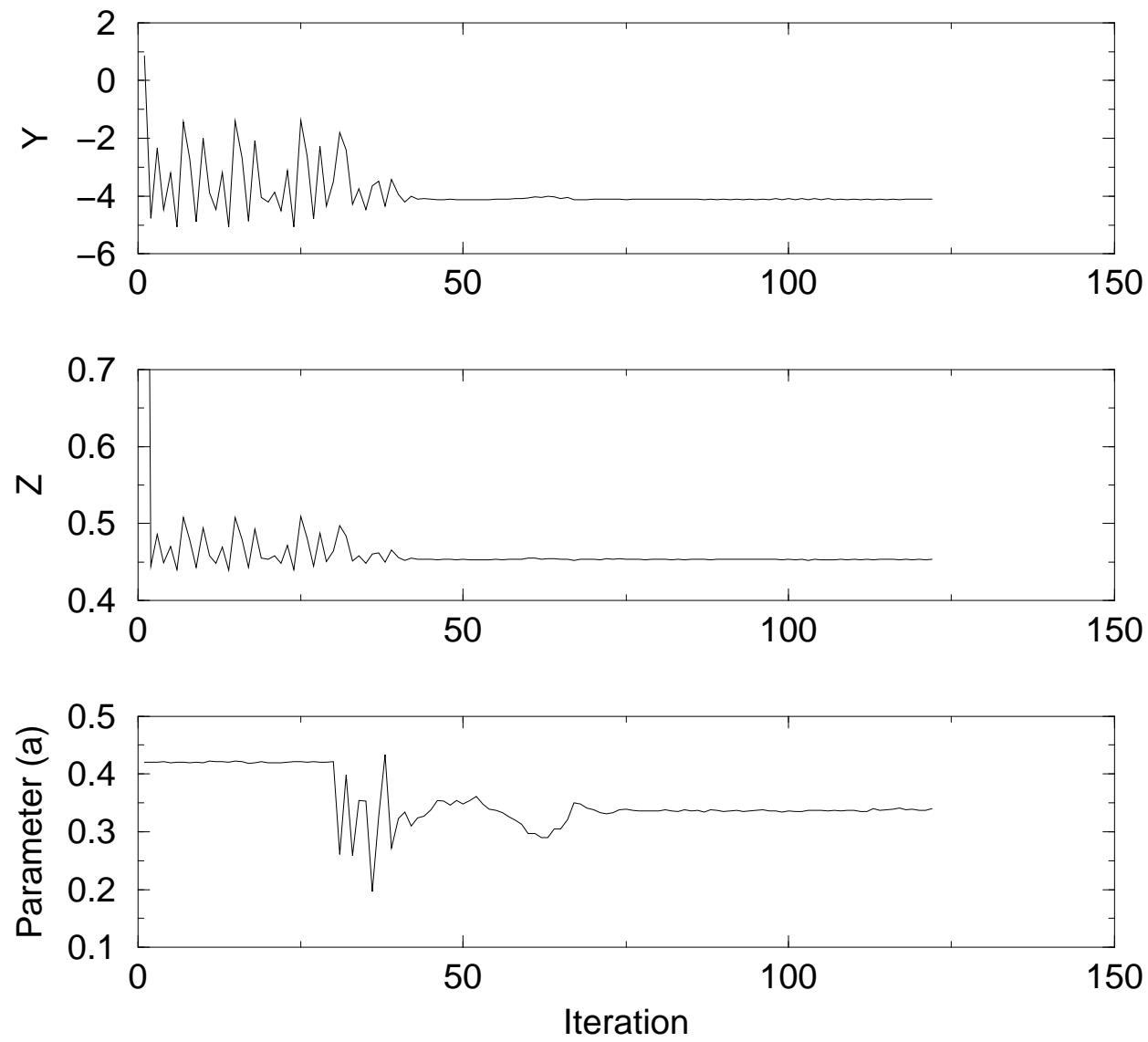

Figure 4: Experimental results for the location of the period doubling bifurcation of the period-1 limit cycle in the Rössler circuit. After a short transient (50 steps) the initial model is properly identified and the circuit is driven to the bifurcation point. The parameters $b$ and $c$ were set at 2.0 and 4.0 respectively. 

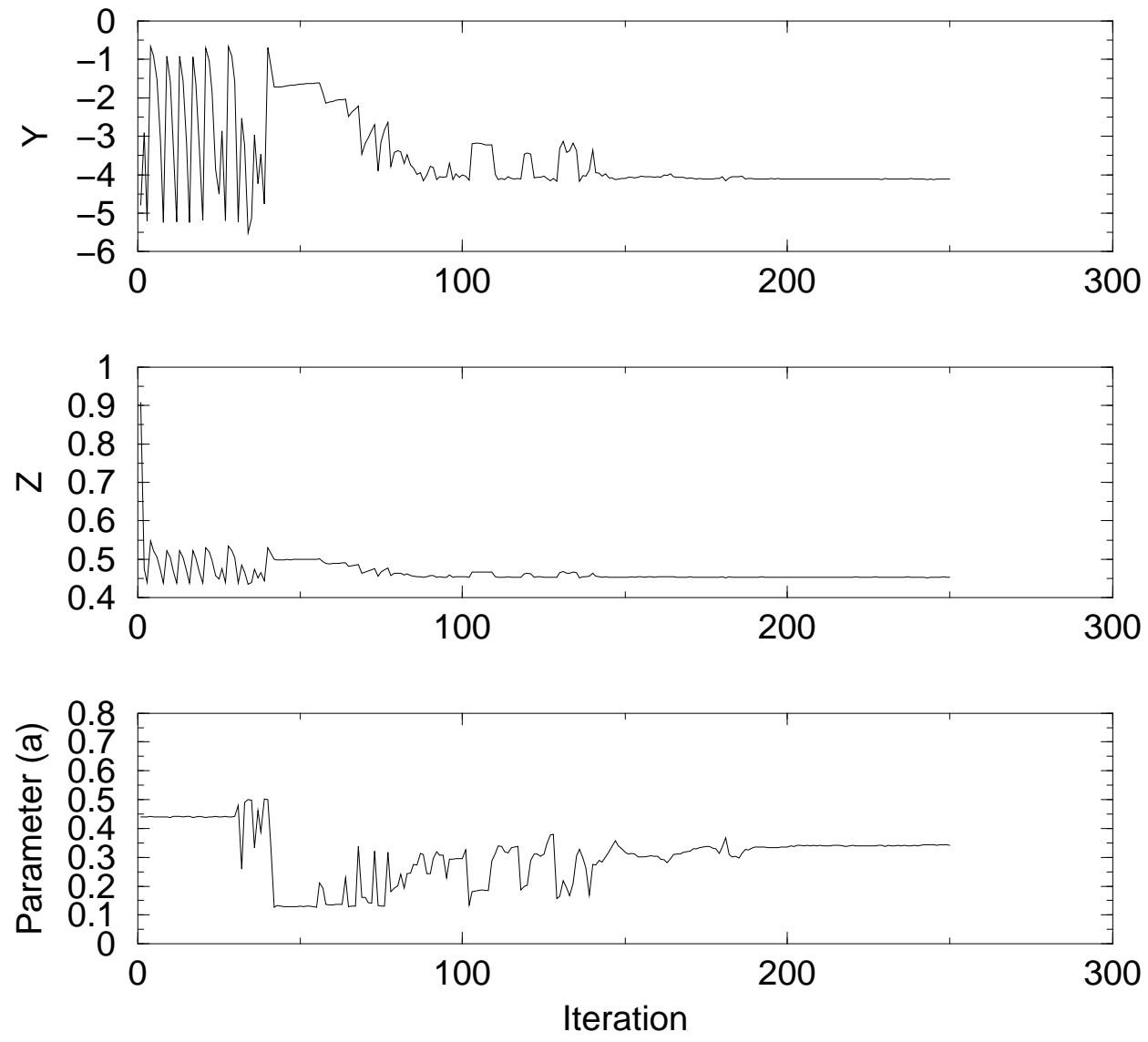

Figure 5: Experimental run exhibiting the effect of the lower bound for the control parameter. The parameter is initially "trapped" near the lower bound until a better model is identified thus restarting the movement towards the bifurcation point. The parameters $b$ and $c$ were set as indicated in Figure 4 . 

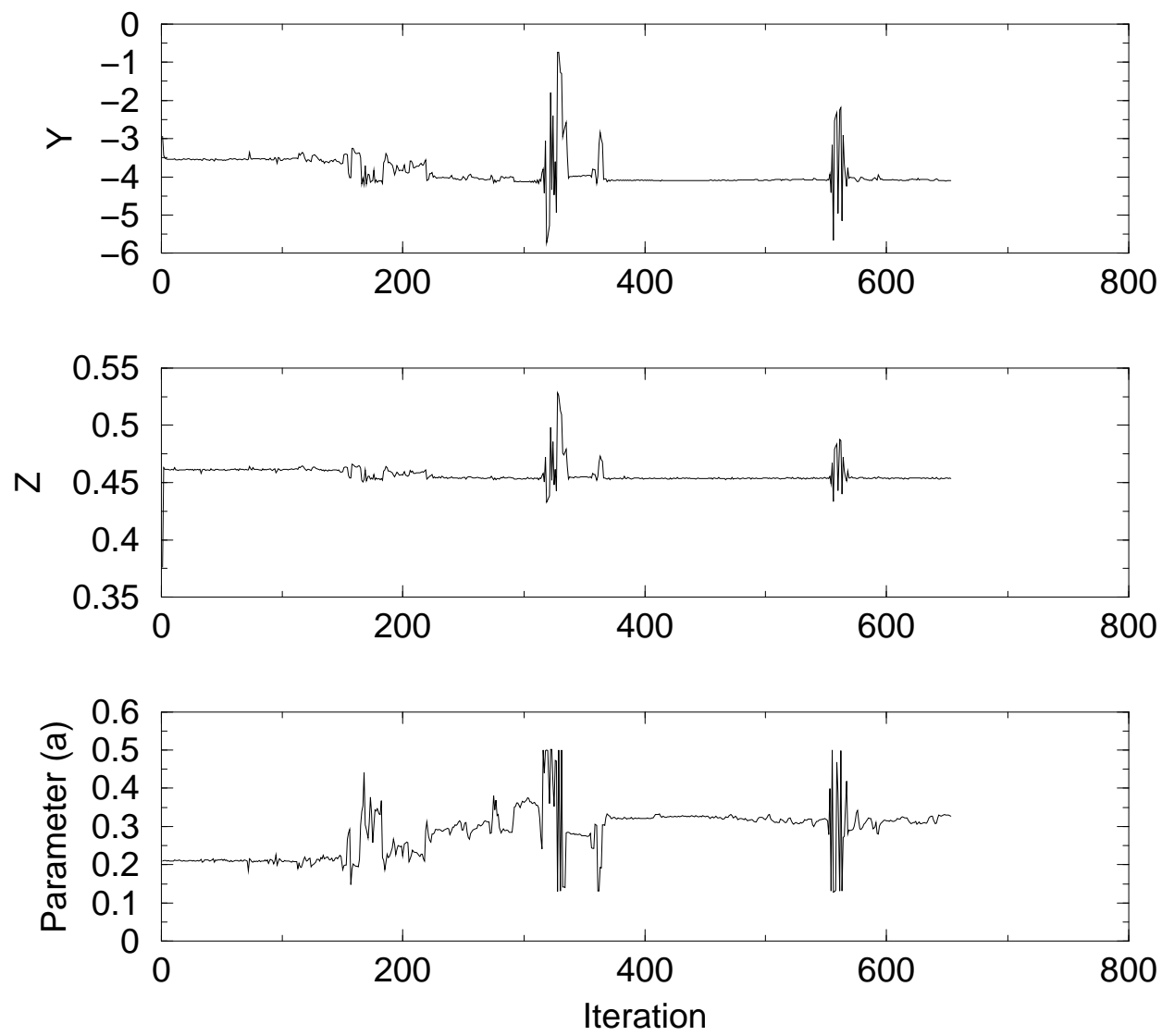

Figure 6: Experimental run exhibiting bursting. The adaptive bifurcation driver settles for a while in a point near the critical coordinates, without achieving convergence, until near-singularity of the matrix used for the identification produces a sudden burst that drives the circuit far away. The parameters $b$ and $c$ were set as indicated in Figure 4. 

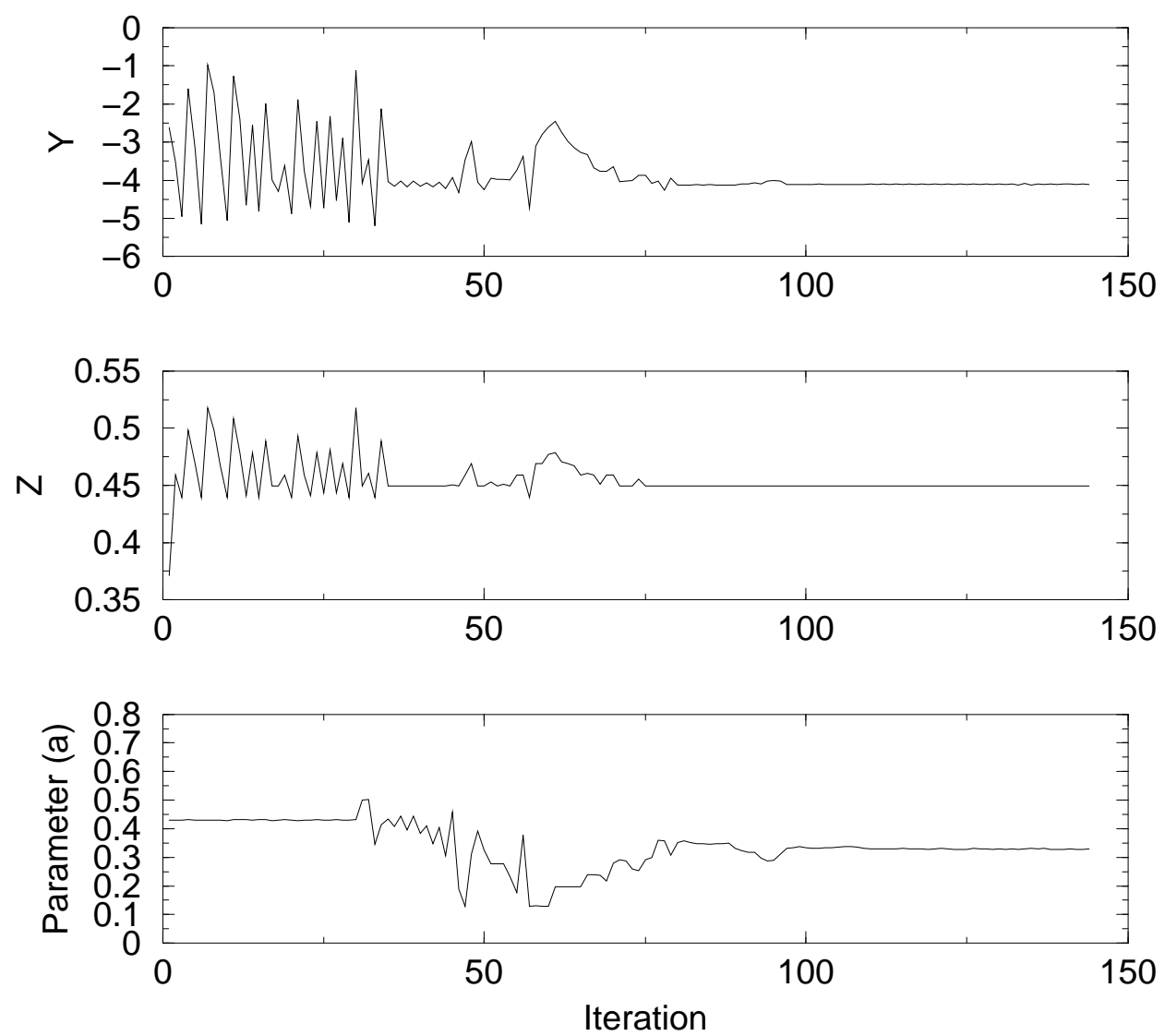

Figure 7: Experimental run with lower resolution. For a resolution based on an 11 bit A/D converter, the bifurcation is also properly located. The parameters $b$ and $c$ were set as indicated in Figure 4. 

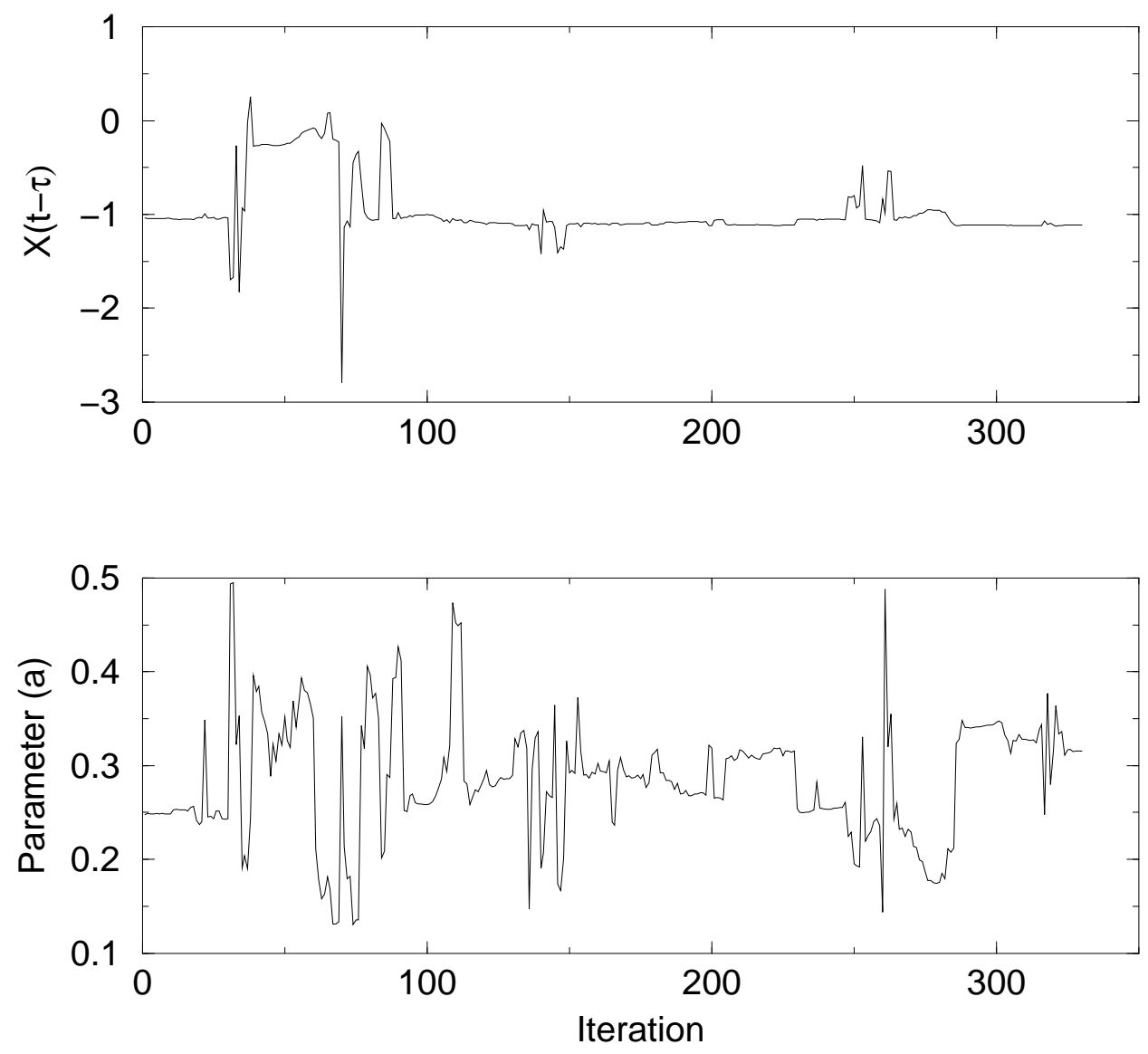

Figure 8: Experimental run using measurements of only one variable and a delay-based reconstruction of the phase-space as the basis to take the Poincaré section. The delay was set to $40 \mathrm{~ms}$, two delays were used and the Poincaré section was set for the current value of the measured variable (x) equal to 0.2. The parameters $b$ and $c$ were set as indicated in Figure 4. 


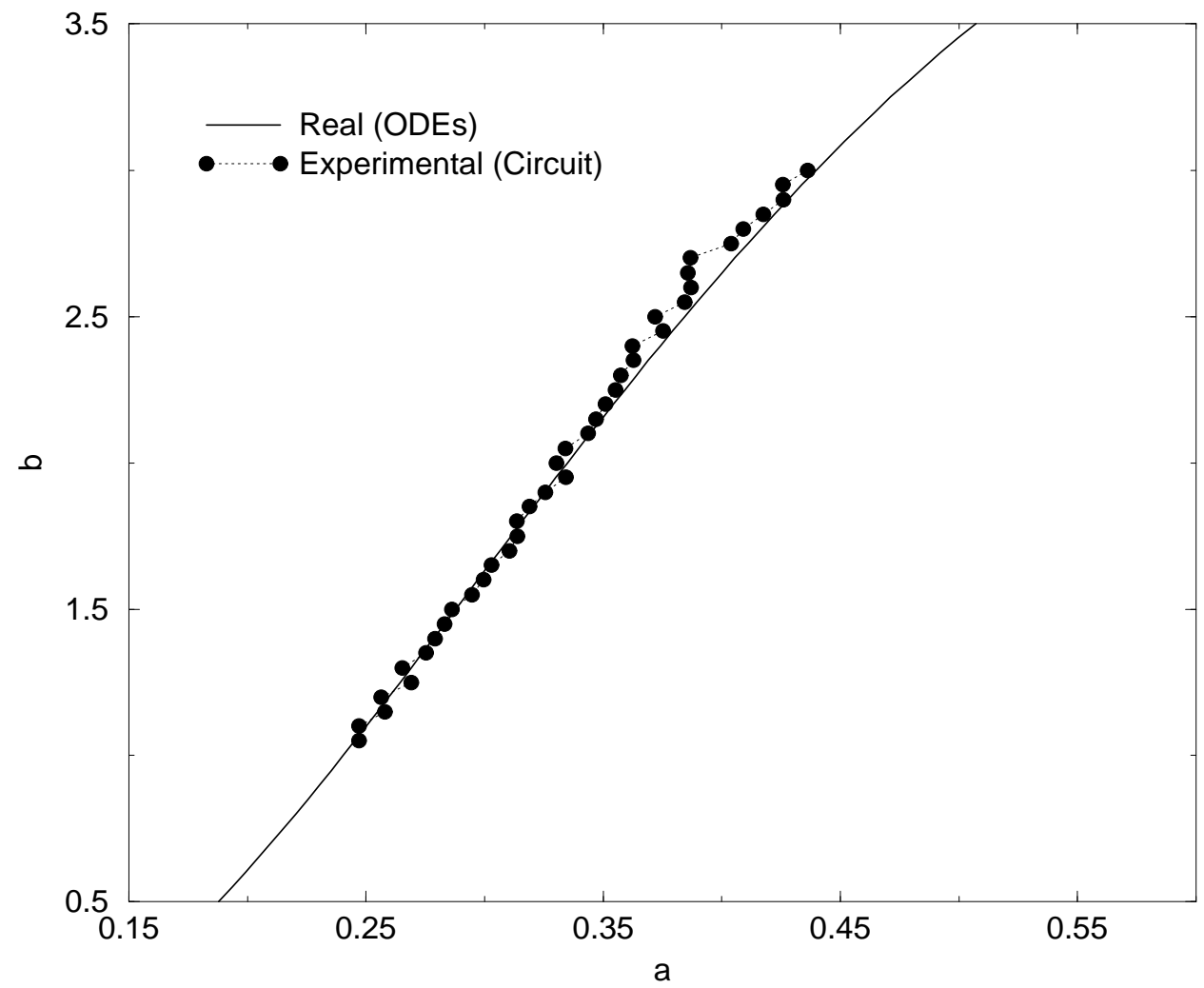

Figure 9: Comparison of the experimentally obtained two parameter boundary and the numerically calculated from the Rössler System. The parameter $b$ was changed gradually and the bifurcation point obtained from the previous value used as starting point to locate the new bifurcation. The experimental results are averaged over 5 runs, three runs using a variation for $b$ decreasing and two for $b$ increasing. The parameter $c$ was set to 4.0 . 


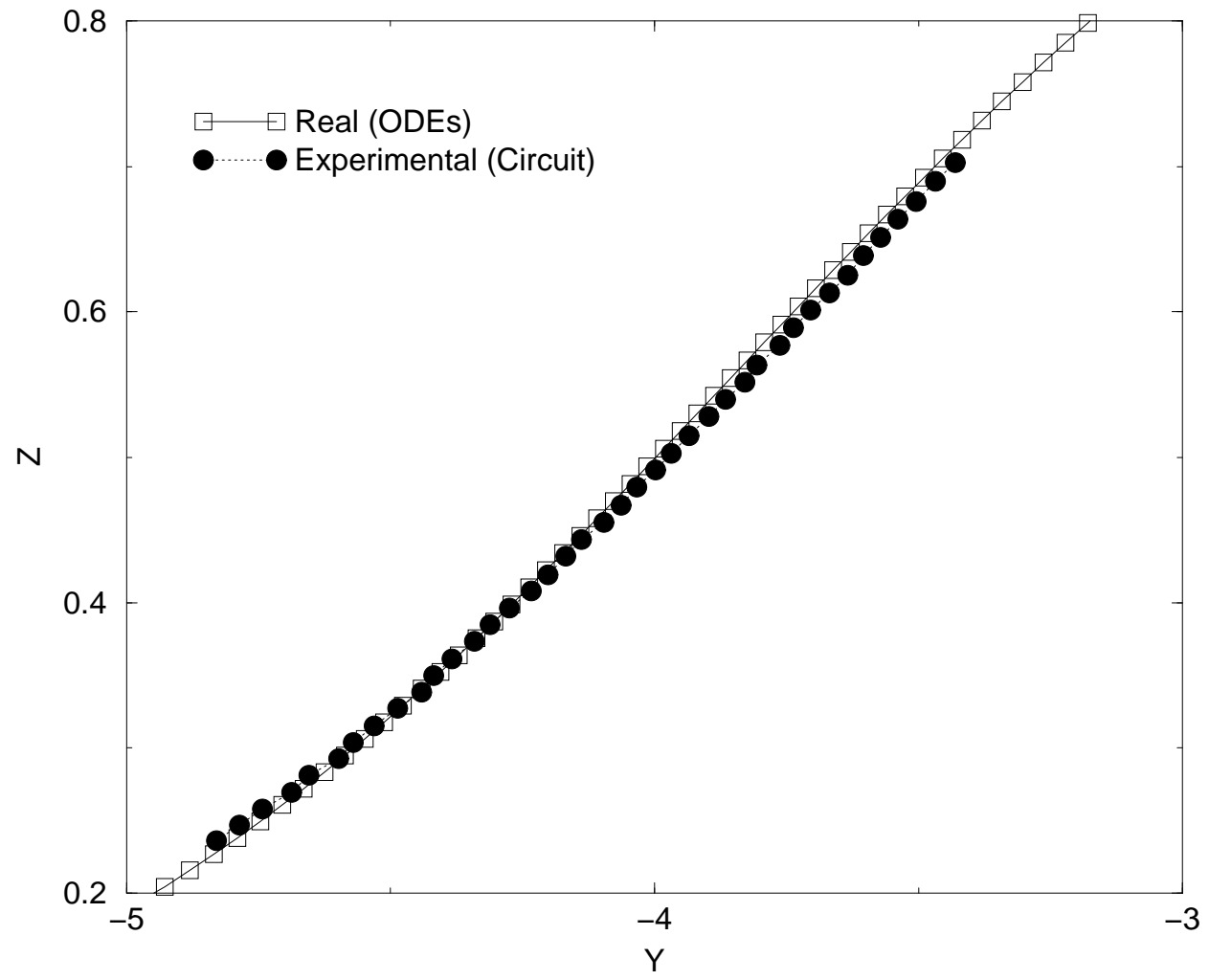

Figure 10: Comparison of the experimentally converged location of the marginally stable fixed points and the numerically calculated ones from the Rössler system. The parameter $b$ was changed as indicated in the previous figure and $c$ was set to 4.0 . 